\title{
Bioadsorción de arsénico del agua del río Locumba utilizando cáscara de naranja (Citrus sinensis), Tacna
}

\author{
Biosorption of arsenic from the Locumba River water using orange peel (Citrus \\ sinensis), Tacna
}

\author{
${ }^{1, *}$ Abad Torres Vargas \\ ${ }^{1}$ Rosmery Choquecota Mena \\ Gabriela Mamani Coaquera \\ ${ }^{1}$ Pedro Ticona Quispe \\ ${ }^{1}$ Marianela Sanga Franco \\ 'Ivon Gutierrez Flores
}

ORCID: 0000-0003-1697-9295

\section{RESUMEN}

En la región Tacna, un problema bastante acentuado es la presencia de As en fuentes de agua superficial, las mismas que son usadas como fuentes de abastecimiento para consumo humano. La presencia de As incrementa los costos de tratamiento, siendo necesaria la búsqueda de tratamientos de menor costo y amigables con el ambiente. En esta investigación, se evaluó la factibilidad del uso de la cáscara de naranja como bioadsorbente para la remoción de As, bajo diferentes tiempos de contacto y temperatura. La muestra de agua utilizada fue del río Locumba. El bioadsorbente se obtuvo luego de un proceso de secado, molienda y tamizado. Con fines de optimización, se aplicó el diseño Box-Behnken, en el que las variables fijas fueron: dosis de bioadsorbente (A), tiempo de contacto (B) y temperatura (C). Las variables que tuvieron efecto en la concentración de As fueron la dosis y tiempo de contacto, alcanzando hasta un $98 \%$ de remoción. En conclusión, la remoción de As del agua del río Locumba aplicando la bioadsorción con la cáscara de naranja es factible y alentadora, aunque hacen falta mayores estudios.

Palabras claves: Box-Behnken, dosis de adsorbente, metales pesados, $\mathrm{pH}$, tiempo de contacto.

\section{ABSTRACT}

In Tacna Region, a significant problem is the presence of As in surface water sources, these are used as water intended for human consumption. The presence of As increased treatment costs, consequently it is necessary to search for low-cost and environmentally-friendly treatments. In this study, the feasibility of using orange peel as biosorption of arsenic was evaluated to As removal under different contact time and temperature. The water sample used was from the Locumba River. The biosorption was obtained after the drying, milling and sieving process. For optimization purposes, Box-Benhnken design was applied with permanent variables: biosorption dose (A) contact time (B) and temperature (C). The variables which had an effect in the concentration of As were biosorption dose and contact time reaching up to $98 \%$ of removal. In conclusion, the As removal of the Locumba River water applying biosorption with orange peel is feasible and encouraging, although more studies are needed.

Key words: Box-Behnken, adsorbent dose, heavy metals, $\mathrm{pH}$, contact time.

${ }^{1}$ Escuela Profesional de Ingeniería Ambiental. Facultad de Ciencias Agropecuarias. Universidad Nacional Jorge Basadre Grohmann. Tacna-Perú, Av. Miraflores S/N.

*Autor de correspondencia: abhadjr@gmail.com 


\section{INTRODUCCIÓN}

La contaminación del agua es un gran problema ambiental que enfrenta la sociedad moderna, que conduce a un desequilibrio ecológico y riesgos para la salud (Feng et al., 2011). Este problema está asociado al inadecuado tratamiento de efluentes domésticos e industriales principalmente. Dentro de los efluentes industriales, uno de los contaminantes que más afectan al medio ambiente son los metales pesados (Nagajyoti et al., 2010). Estos están considerados como uno de los grupos más peligrosos debido a su baja biodegradabilidad, su alta toxicidad a bajas concentraciones y su capacidad de bioacumulación (CañizaresVillanueva, 2000). Los métodos convencionales para la eliminación de metales del agua incluyen reducción, precipitación, intercambio iónico, reducción electroquímica y ósmosis inversa (e.g. (Semerjian y Ayoub, 2003; Lee et al., 2007; -Rajasulochana y Preethy, 2016). La mayoría de ellos implican altos costos de capital con gastos recurrentes, que no son adecuados para las industrias de pequeña escala (Moreno-Piraján y Giraldo, 2012).

Recientemente se está desarrollado tecnologías más amigables con el ambiente y más económicas. Dentro de estas se encuentra la biosorción, que es un proceso fisicoquímico que incluye los fenómenos de adsorción y absorción de moléculas e iones (Tejada-Tovar et al., 2015). Este método es de fácil acceso, bajo costo y tiene gran capacidad de remoción de los metales pesados (Tejada-Tovar et al., 2015). Particularmente, la bioadsorción promueve el uso de materiales naturales renovables de bajo costo, que poseen características biosorbentes y ha mostrado ser muy eficiente en la remoción de diversos contaminantes del agua (Wang y Chen, 2009; Aguilar y Flores, 2018). Las paredes celulares de los materiales bioadsorbentes contienen polisacáridos, proteínas y lípidos, y, por lo tanto, numerosos grupos funcionales capaces de enlazar metales pesados en la superficie de estos. Entre los grupos funcionales presentes se pueden mencionar los grupos amino, carboxílico, hidroxílico, fosfato y tiol que difieren en su afinidad y especificidad respecto a la susceptibilidad para unirse a diferentes iones metálicos(Ghimire et al., 2003).

Entre los materiales que presentan una adecuada capacidad de adsorción se pueden mencionar a la cáscara de yuca (Tejada et al., 2016), la cáscara de plátano (Annadurai et al., 2003) y la cáscara de naranja (Feng et al., 2011; Pardo et al., 2018). La cáscara de naranja tiene un alto contenido de carbono $(42.70 \%)$, presencia de mesoporosos (Garcia y Granillo, 2017) y alto contenido de celulosa, pectina, hemi-celulosa, lignina y pigmentos de clorofila que contienen muchos grupos funcionales hidroxilo (Bhatnagar et al. 2015), haciéndola un bioadsorbente para muchos contaminantes. Por ejemplo, ha sido utilizada exitosamente para la remoción de $\mathrm{Cd}, \mathrm{Pb}$ y $\mathrm{Ni}$ (Feng et al., 2011; Moreno-Piraján y Giraldo, 2012; -Cardona-Gutiérrez et al., 2013), Zn, Co, $\mathrm{Cu}$ (Annadurai et al., 2003; Moreno-Piraján y Giraldo, 2012; -Cardona-Gutiérrez et al., 2013) y As (Khaskheli et al., 2011; Peng et al., 2013; Abid et al., 2016).

En Perú, el número de personas expuestas a concentraciones de As es superior a los niveles recomendados, en el agua excede los $250 \mathrm{mg} / \mathrm{L}$. Aun cuando los casos de contaminación reportados se dan en varias regiones del país, los más relevantes se presentan en los departamentos del sur, relacionados con la actividad volcánica de la zona. En la región Tacna, la presencia de As en el agua de consumo humano es un problema condicionado esencialmente por su ubicación geográfica, en suelos volcánicos que generan la contaminación natural de aguas geogénicas por la disolución del As (INGEMMET, 2016). En la región Tacna, 19 de los 27 distritos (70\%) exceden los límites máximos permisibles de As en agua de consumo humano establecido por la OMS y la legislación peruana (MINSA, 2017), encontrándose concentraciones tóxicas de As en la orina (Ale-Mauricio et al., 2018). Entonces, es necesaria la búsqueda de alternativas sostenibles para la remoción de As del agua. De este modo, el principal objetivo fue evaluar la factibilidad de uso de la cáscara de naranja como bioadsorbente para la remoción de As, bajo diferentes tiempos de contacto y temperatura.

\section{MATERIALY MÉTODOS}

\section{Colección de muestras}

La muestra de agua se tomó del río Locumba, distrito de Locumba, departamento de Tacna, entre las coordenadas $17^{\circ} 37^{\prime} 04^{\prime \prime} \mathrm{S}$ y $70^{\circ} 45^{\prime} 38^{\prime \prime} \mathrm{O}$ y a $571 \mathrm{~m} \mathrm{s.n.m}$. Se tomó una muestra simple de $7 \mathrm{~L}$ de la parte central del río, siguiendo el protocolo 
establecido por ANA (2016). La muestra fue analizada para determinar la concentración inicial de As; así como, el nivel de $\mathrm{pH}$ y conductividad eléctrica (CE).

\section{Preparación del material bioadsorbente}

La cáscara de naranja se obtuvo a partir de vendedores de jugo de fruta de mercados locales. La cáscara fue lavada varias veces para remover cualquier impureza. Luego, fue sometida a un proceso de secado bajo la exposición del sol durante tres días. Posteriormente, se trituró $60 \mathrm{~g}$ de cáscara seca hasta lograr su pulverización.
Finalmente, la muestra fue tamizada con el fin de obtener una muestra homogénea.

\section{Diseño de la investigación}

Se aplicó un diseño de Box-Behnken. Las variables fueron dosis de bioadsorbente (A), tiempo de contacto (B) y temperatura (C). Todas las variables tuvieron tres niveles conteniendo el valor mínimo, intermedio y máximo. Se obtuvieron 15 corridas, de las cuales tres fueron repeticiones del punto central. Para cada corrida se utilizaron $250 \mathrm{ml}$ de agua. Los análisis se realizaron en el Software Statgraphics.Tabla 1.

Tabla 1. Variables independientes y diferentes niveles usados en MSR

\begin{tabular}{lcccc}
\hline \multirow{2}{*}{ Factores } & Código & \multicolumn{3}{c}{ Niveles de las variables } \\
& & -1 & 0 & +1 \\
\hline Dosis de bioadsorbente $(\mathrm{g})$ & $\mathrm{A}$ & 1 & 4 & 7 \\
Tiempo de contacto (día) & $\mathrm{B}$ & 1 & 4 & 7 \\
Temperatura $\left({ }^{\circ} \mathrm{C}\right)$ & $\mathrm{C}$ & 18 & 24 & 30 \\
\hline
\end{tabular}

\section{Análisis de variables}

Por cada corrida, se analizaron el $\mathrm{pH}$, la CE y la concentración de As. La medición del pH y la CE se realizaron con un multiparámetro HQ40d, y la concentración de As con el método AWWA 3114-C "Equipo de absorción atómica y generador Hidruros Marca Thermo Scientific ICE 3000 Series. Los análisis se realizaron en el laboratorio de la EPS Tacna.

\section{RESULTADOS}

Los niveles iniciales de $\mathrm{pH}, \mathrm{CE}$ y As de la muestra de agua del río Locumba estuvieron por encima de los límites máximos permisibles para consumo humano (Tabla 2).

Tabla 2. Características de la muestra inicial del agua del río Locumba

\begin{tabular}{cccc}
\hline \multirow{2}{*}{ Parámetros } & Unidad & Muestra del río Locumba & $\begin{array}{c}\text { Norma Consumo Humano D.S. 031- } \\
\text { 2010-SA }\end{array}$ \\
\hline $\mathrm{pH}$ & und & 8.29 & $6.5-8.5$ \\
$\mathrm{CE}$ & $\mu \mathrm{S} / \mathrm{cm}$ & 2200 & 1500 \\
$\mathrm{As}$ & $\mathrm{mg} / \mathrm{L}$ & 0.450 & 0.010 \\
\hline
\end{tabular}

Luego de la aplicación de la cáscara de naranja como bioadsorbente, se obtuvo una remoción de hasta 98 \%, esto aplicando una dosis de $4 \mathrm{~g}, 4$ días de contacto y a $24^{\circ} \mathrm{C}$ (Tabla 3 ). 
Tabla 3. Resultados de pH, conductividad eléctrica y concentración de arsénico.

\begin{tabular}{ccccccr}
\hline $\begin{array}{c}\text { Dosis de } \\
\text { Bioadsorbente }\end{array}$ & $\begin{array}{c}\text { Tiempo de } \\
\text { contacto }\end{array}$ & Temperatura & $\mathrm{pH}$ & $\mathrm{CE}$ & $\begin{array}{c}\text { Concentración } \\
\mathrm{de} \text { As }\end{array}$ & $\begin{array}{c}\text { \% de } \\
\text { Remoción }\end{array}$ \\
\hline 1 & 1 & 24 & 4.55 & 2390 & 0.05 & 88.889 \\
7 & 1 & 24 & 3.82 & 3490 & 0.03 & 93.333 \\
1 & 7 & 24 & 4.22 & 2470 & 0.05 & 88.889 \\
7 & 7 & 24 & 4.25 & 3130 & 0.03 & 93.333 \\
1 & 4 & 18 & 4.47 & 2350 & 0.045 & 90.000 \\
7 & 4 & 18 & 4.16 & 3310 & 0.025 & 94.444 \\
1 & 4 & 30 & 4.24 & 2360 & 0.03 & 93.333 \\
7 & 4 & 30 & 4.11 & 3320 & 0.02 & 95.556 \\
4 & 1 & 18 & 3.81 & 3010 & 0.04 & 91.111 \\
4 & 7 & 18 & 4.11 & 2990 & 0.025 & 94.444 \\
4 & 1 & 30 & 4.19 & 2840 & 0.06 & 86.667 \\
4 & 7 & 30 & 4.01 & 2990 & 0.02 & 95.556 \\
4 & 4 & 24 & 4.17 & 2840 & 0.035 & 92.222 \\
4 & 4 & 24 & 4.09 & 2830 & 0.05 & 88.889 \\
4 & 4 & 24 & 4.17 & 2840 & 0.01 & 97.778 \\
\hline
\end{tabular}

Los modelos de regresión reducidos significativos se presentan en las Ecuaciones 1, 2 y 3, considerando un nivel de significancia del 0.1. En ningún caso la temperatura tuvo un efecto significativo. El pH se relacionó negativamente con la dosis de cáscara de naranja $(\mathrm{F}=3.47, \mathrm{p}=$ 0.054) (Figura 1), al contrario de la CE que se relacionó en forma positiva $(\mathrm{F}=79.92, \mathrm{p}<$ 0.0001) (Figura 2). La concentración de As se relacionó de forma negativa, tanto con la dosis como con el tiempo de contacto $(\mathrm{F}=3.33, \mathrm{p}=$ 0.0705) (Figura 3). Esto significa que a mayor dosis y tiempo de contacto hay una mayor remoción de As. Los coeficientes de determinación $\left(\mathrm{R}^{2}\right)$ fueron $0.49,0.93$ y 0.36 para el pH, CE y As respectivamente. El modelo que mejor se ajustó fue el de la CE, ya que el modelo explicó el $93 \%$ de su variabilidad. En cambio, para el caso del $\mathrm{pH}$ y As $\mathrm{R}^{2}$ fue inferior a 0.5. Además, los coeficientes de determinación ajustados fueron $0.35,0.92,0.25$ para el $\mathrm{pH}, \mathrm{CE}$ y As respectivamente.

$$
\begin{aligned}
& \mathrm{pH}=-18.50-0.20 \mathrm{~A}+0.02 \mathrm{~A}^{2} \\
& \mathrm{CE}=3301.09+153.09 \mathrm{~A} \\
& \mathrm{As}=0.06-0.0029 \mathrm{~A}-0.0023 \mathrm{~B}
\end{aligned}
$$
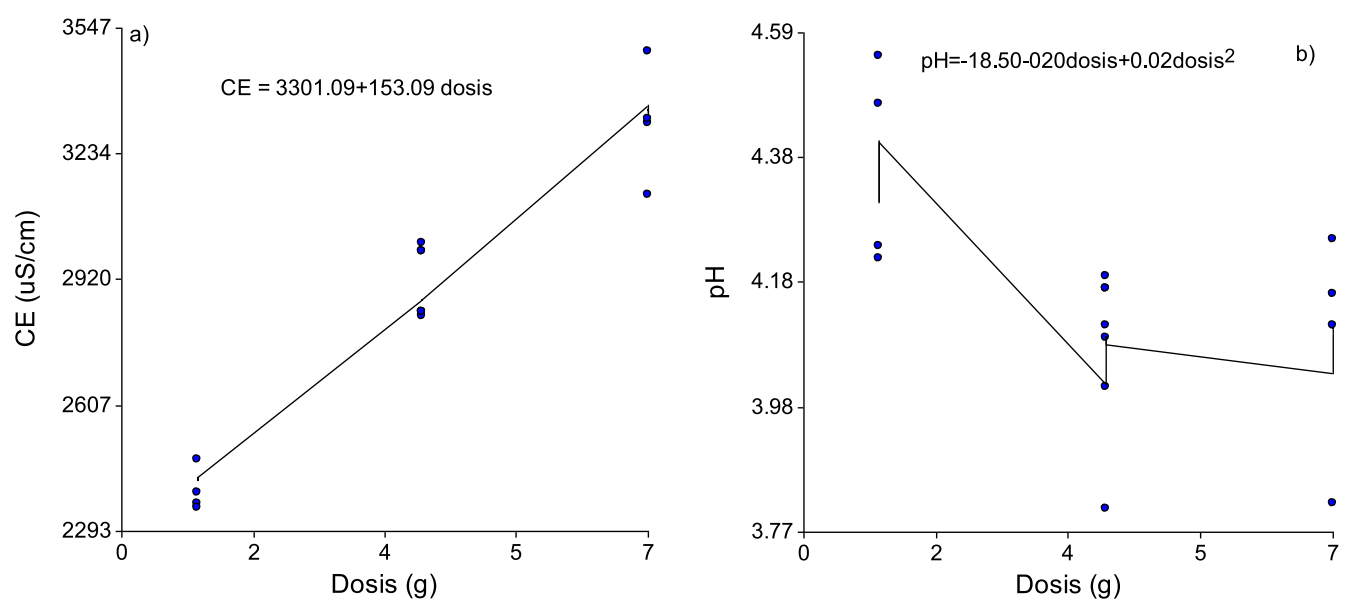

Figura 1. Relación entre la CE y dosis de cáscara de naranja (a) y del pH con la dosis de cáscara de naranja (b 


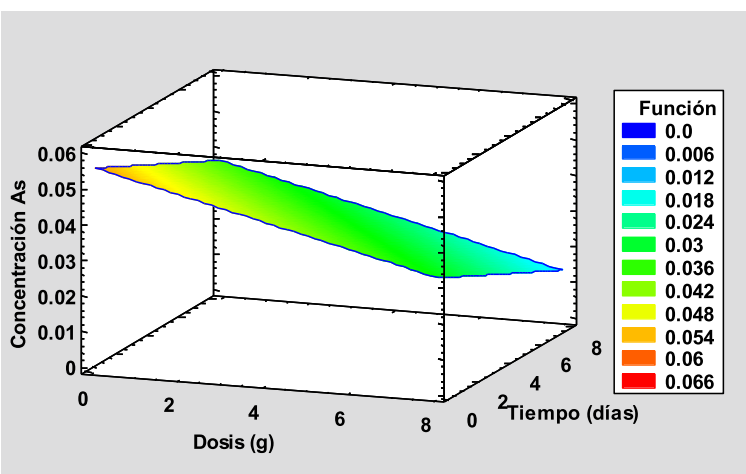

Figura 2. Superficie de respuesta de la concentración de As (mg/l) con relación a la dosis de cáscara de naranja (g) y el tiempo de contacto (días) al bioadsorbente.

\section{DISCUSIÓN}

Los niveles de remoción de As estimados fueron altos $(>85 \%)$ en los diferentes niveles de dosis, tiempo de contacto y temperatura, aunque este último no tuvo un efecto significativo. El nivel de remoción encontrado fue similar a lo reportado utilizando cáscara de banano (Caballero, 2012) y cáscara de naranja pre-tratada (e.g. Khaskheli et al., 2011; Peng et al., 2013; Abid et al., 2016). Tanto la dosis como el tiempo de contacto con el bioadsorbente tuvieron un efecto positivo en la disminución de la concentración de As. Esto debido a que el incremento de la dosis del adsorbente incrementa los sitios de ligandos (Khaskheli et al., 2011), contribuyendo a una mayor bioadsorción. Sin embargo, es de esperar que exista un punto de inflexión, a partir del cual el incremento de la dosis tenga un efecto negativo (Ajaykumar et al., 2009), debido al bloqueo de los poros y sitios de adsorción y/o incremento en la competencia de ligandos cargados negativamente (Abid et al., 2016). Del mismo modo, la tasa de adsorción según el tiempo de contacto tiene un punto de inflexión, a partir del cual la tasa de adsorción es muy lenta o no significativa (e.g. Aoyama et al., 2000; Ghimire et al., 2003; Moreno-Piraján y Giraldo 2012). En esta investigación no pudo detectarse el punto de inflexión con respecto al tiempo de contacto, debido al prolongado tiempo de contacto considerado (1, 4 y 7 días). La curva de estabilización de la adsorción de metales pesados ocurre hasta dentro de las primeras $72 \mathrm{~h}$ (Aoyama et al., 2000; Ghimire et al., 2003), variando según el metal y las condiciones experimentales. Entonces, hizo falta disminuir el rango de evaluación del tiempo de contacto, pero aumentando los niveles de evaluación para detectar el punto de estabilización de la bioadsorción del As. Particularmente, en el caso de 7 días de tiempo de contacto, pudo haber ocurrido al menos un proceso de desorciónreadsorción; ya que, la cáscara de naranja no pierde sus propiedades de bioadsorción hasta en dos o tres veces de reutilización (Annadurai et al., 2003; Feng et al., 2011).

Aunque el $\mathrm{pH}$ es un factor determinante en el proceso de adsorción (Annadurai et al., 2003; Feng et al., 2011; Peng et al., 2013; Abid et al., 2016), no fue un factor controlado durante el experimento. Por el contrario, fue considerado como una variable respuesta. De este modo, el pH disminuyó conforme se incrementó la dosis de bioadsorbente, similar a lo reportado por Ccencho (2018). El pH inicial del agua del río Locumba fue 8.29 , siendo muy alto para un adecuado proceso de bioadsorción de As (Ghimire et al. 2003; Ajaykumar et al., 2009; Tejada-Tovar et al., 2015). En general, para la adsorción de cationes metálicos como el As, los valores óptimos de $\mathrm{pH}$ fluctúan alrededor de 4 (e.g. Ajaykumar et al., 2009; Moreno-Piraján y Giraldo, 2012). Valores muy ácidos, por debajo de 3 , implican una baja adsorción debido a la alta competencia de $\mathrm{H}+$ con los cationes metálicos (Feng et al., 2011). En cambio, con valores altos de $\mathrm{pH}$ (superiores a 5), los metales podrían precipitar en forma de hidróxidos (Aoyama et al., 2000; Moreno-Piraján y Giraldo, 2012), siendo muy baja la remoción por bioadsorción propiamente. En este estudio, con la adición de la cáscara de naranja, el pH se disminuyó entre 3.81 a 4.55 ; de modo que, se posibilitó la bioadsorción de As. Por otra parte, aunque muchos estudios indican la influencia de la temperatura en el proceso de adsorción (e.g. Aoyama et al., 2000; Ajaykumar et al., 2009; Tejada-Tovar et al., 2015), en este estudio no se pudo detectar su influencia significativa en ninguna de las variables evaluadas, probablemente debido al bajo control que se tuvo de la misma.

Los resultados de este estudio son preliminares, pero alentadores, evidenciando la factibilidad del uso de la cáscara de naranja como un adsorbente del As del agua del río Locumba. Esta tecnología es costo-efectivo y amigable con el ambiente (Peng et al., 2013), de forma que se reduciría los costos de tratamiento del agua en la región de Tacna. Sin embargo, hacen falta mayores estudios, por ejemplo la determinación de isotermas de adsorción para describir cómo las 
moléculas o iones del adsorbato interactúan con la superficie del sorbente y su grado de acumulación en la superficie del sorbente (Peng et al., 2013) o para determinar la eficiencia de adsorción (Khaskheli et al., 2011). Además, con fines de incrementar la bioadsorción, la cáscara de naranja podría ser pre-tratada para incrementar la superficie de adsorción (Aoyama et al., 2000; Abid et al., 2016).

\section{CONCLUSIÓN}

Se determinó la eficiencia de remoción de As del agua del río Locumba con la cáscara de naranja, encontrándose altos niveles de remoción (98\%). Tanto la dosis del adsorbente como el tiempo de contacto tuvieron un efecto importante en la remoción del As. Respecto a la $\mathrm{CE}$ y pH, solo la dosis de adsorbente tuvo un efecto significativo.

\section{AGRADECIMIENTOS}

A la Empresa Prestadora de Servicios -Tacna S.A. por brindar las facilidades de análisis de parámetros en su laboratorio.

\section{REFERENCIAS}

Abid, M.; Niazi, N.K.; Bibi, I.; Farooqi, A.; Sik, Y.; Kunhikrishnan, A.; Ali, F.; Ali, S.; Igalavithana, A.D. \& Arshad, M. (2016). Arsenic (V) biosorption by charred orange peel in aqueous environments. Int $J$. Phytoremediation, 18, 442-449. doi: 10.1080/15226514.2015.1109604

Aguilar, M.B. y Flores, C.P. (2018). Evaluación de la cáscara de naranja como material adsorbente natural de ion metalico $\mathrm{Cu}(\mathrm{II})$ (tesis de pregrado). Universidad Nacional de San Agustin, Arequipa.

Ajaykumar, A.V.; Darwish, N.A. y Hilal, N. (2009). Study of various parameters in the biosorption of heavy metals on activated sludge. World Applied Sciences Journal, 5, 32-40.

Ale-Mauricio, D.A.; Villa G. y Gastañaga M. (2018). Concentración de arsénico urinario en pobladores de dos distritos de la región Tacna Perú, 2017. Rev Peru Med Exp Salud Pública, 35(2), 183-189. doi: 10.17843/rpmesp.2018.352.3693.183

Annadurai, G.; Juang, R.S. \& Lee D.J. (2003). Adsorption of heavy metals from water using banana and orange peels. Water Sci Technol 47:185-190. doi: 10.2166/wst.2003.0049

Aoyama, M.; Tsuda M., Cho, N.S. \& Doi, S. (2000). Adsorption of trivalent chromium from dilute solution by conifer leaves. Wood Sci Technol, 34, 55-63. doi: $10.1007 / \mathrm{s} 002260050008$

Autoridad Nacional del Agua [ANA]. (2016). Protocolo Nacional para el Monitoreo de la Calidad de los Recursos Hídricos Superficiales. Gráfica Industrial Alarcón S.R.L. Resolución Jeatural N ${ }^{\circ}$ 010-2016ANA. 92pp.

Bhatnagar, A.; Sillanpää, M. \& Witek-Krowiak, A. (2015). Agricultural waste peels as versatile biomass for water purification - A review. Chem Eng $J$ 270, 244-271. doi: 10.1016/j.cej.2015.01.135

Caballero, V.M. (2012). Evaluación del uso de la cáscara de banano (Musa AAA) variedad Williams para la remoción de arsénico en el agua para consumo humano (tesis de pregrado). Universidad de San Carlos de Guatemala.

Cañizares-Villanueva R.O. (2000). Biosorción de metales pesados mediante el uso de biomasa microbiana. Rev Latinoam Microbiol, 42, 131-143

Cardona-Gutiérrez, A.F.; Cabañas-Vargas, D.D. y Zepeda-Pedreguera, A. (2013). Evaluación del poder biosorbente de cáscara de naranja para la eliminación de metales pesados, $\mathrm{Pb}$ (II) y Zn (II). Ingeniería 17:1-9.

Ccencho, S. (2018). Uso de biomasa seca (cáscara de plátano) como bioadsorbente de arsénico en agua subterránea, Cruz del Médano, Mórrope, Lambayeque, a nivel laboratorio - 2018 (tesis de pregrado). Universidad César Vallejo, Lima.

Feng, N.; Guo, X.; Liang, S.; Zhu, Y. \& Liu, J. (2011). Biosorption of heavy metals from aqueous solutions by chemically modified orange peel. $J$ Hazard Mater, 185, 49-54. doi: 10.1016/j.jhazmat.2010.08.114

Garcia, R.A. y Granillo, Y. (2017). Evaluacion de las condiciones operacionales en el proceso de prepratacion de carbon activo de cascara de naranja (Citrus Sinensis Linn Osbeck) (tesis de pregrado). Universidad Autonoma de Nicaragua.

Ghimire, K.N.; Inoue, K.; Yamaguchi, H.; Makino, K. y Miyajima, T. (2003). Adsorptive separation of arsenate and arsenite anions from aqueous medium by using orange waste. Water Res $37: 4945-4953$. d o i : 
10.1016/j.watres.2003.08.029

Instituto Geológico Minero y Metalúrgico [INGEMMET]. (2016). Zonas críticas por peligros geológicos en la región de Tacna. Informe técnico. 52pp.

Khaskheli, M.I.; Memon, S.Q., Siyal, A.N. \& Khuhawar, M.Y. (2011). Use of orange peel waste for arsenic remediation of drinking water. Waste and Biomass Valorization, 2, 423-433. doi: 10.1007/s12649-011-9081-7

Lee, M., Paik, I.S., Kim, I.; Kang, H. \& Lee, S. (2007). Remediation of heavy metal contaminated groundwater originated from abandoned mine using lime and calcium carbonate. J Hazard Mater, 144, 208-214. doi: 10.1016/j.jhazmat.2006.10.007

Ministerio de Salud [MINSA]. (2017). Análisis de situación de salud, región Tacna. Informe técnico de la Dirección Regional de Salud Tacna. 114 pp.

Moreno-Piraján, J.C. y Giraldo, L. (2012). Heavy metal ions adsorption from wastewater using activated carbon from orange peel. $E$ Journal Chem, 9, 926-937. doi: $10.1155 / 2012 / 383742$

Nagajyoti, P.C.; Lee, K.D. \& Sreekanth, T.V.M. (2010). Heavy metals, occurrence and toxicity for plants: A review. Environ Chem Lett, 8, 199-216. doi: 10.1007/s10311-0100297-8

Pardo, K.; Cogley, K.; Cisneros, A.; De Gracia, S. y Tejedor de León A. (2018). Aprovechamiento de las cáscaras de la naranja como bioabsorbente para el tratamiento de efluentes industriales. Rev Iniciación Científica, 4, 9-14. doi: 10.33412/rev-ric.v4.0.1812

Peng ,Y.; Xiao; H.Y.; Cheng, X.Z. \& Chen H.M. (2013). Removal of arsenic from wastewater by using pretreating orange peel. Adv Mater Res, 773, 889-892. doi: 10.4028/www.scientific.net/AMR.773.889

Rajasulochana P. \& Preethy V. (2016). Comparison on efficiency of various techniques in treatment of waste and sewage water - A comprehensive review. Resour Technol, 2, 175-184. doi : 10.1016/j.reffit.2016.09.004

Semerjian, L. \& Ayoub, G. (2003). High pH magnesium coagulation flocculation in wastewater treatment. Adv Environ Res, 7, 389-403.

Tejada-Tovar, C.; Villabona-Ortiz, Á. y GarcésJaraba, L. (2015). Adsorción de metales pesados en aguas residuales usando materiales de origen biológico. Tecno Lógicas, 18, 109-123. doi : 10.22430/22565337.209

Tejada, C.N.; Montiel, Z. y Acevedo, D. (2016). Aprovechamiento de cáscaras de Yuca y Ñame para el tratamiento de aguas residuales contaminadas con $\mathrm{Pb}(\mathrm{II})$. Inf Tecnol, 27, 9-20. doi: 10.4067/S071807642016000100003

Wang, J. \& Chen, C. (2009). Biosorbents for heavy metals removal and their future. Biotechnol Adv, 27, 195-226. doi: 10.1016/j.biotechadv.2008.11.002 